\title{
CPPD crystal deposition disease in patients with rheumatoid arthritis
}

Received: 26 November 2004 / Revised: 28 April 2005 / Accepted: 28 April 2005 / Published online: 20 December 2005

(C) Clinical Rheumatology 2005

\begin{abstract}
The aim of this study was to assess the frequency and the outcome of patients suffering from rheumatoid arthritis in which calcium pyrophosphate dihydrate (CPPD) crystal deposits were found to coexist in synovial fluid analysis. Such association was more frequent than previously believed with CPPD crystals found in $25.8 \%$ of 93 patients with rheumatoid arthritis. As a group, a trend toward a worse outcome was suggested by more frequent prostheses of the lower limb.
\end{abstract}

Keywords CPPD crystal deposition diseases $\cdot$ Joint prosthesis $\cdot$ Rheumatoid arthritis

\section{Introduction}

In osteoarthritis (OA), severe joint lesions were observed more frequently in cases in which pyrophosphate arthropathy coexists [1]. It has also been shown that calcium pyrophosphate dihydrate (CPPD) crystal deposition disease is present in about $20 \%$ of patients suffering from OA [2].

By contrast, CPPD crystal deposition disease has been considered to be very rare in rheumatoid arthritis (RA) [3]. As secondary OA may be superimposed to an old rheumatoid damage, it appeared interesting to determine the frequency with which CPPD crystal deposits were found in synovial fluid (SF) of RA patients and to compare the clinical outcome of RA patients with and without CPPD crystals in the SF.

J. C. Gerster $(\bowtie) \cdot$ J. Kern · J. Dudler · A. K. L. So

Department of Rheumatology, CHUV,

1011 Lausanne, Switzerland

e-mail: Jean-Charles.Gerster@chuv.hospvd.ch

P. A. Varisco

Department of Medicine, Kantonsspital,

6000 Lucerne 16, Switzerland

\section{Patients and methods}

In a retrospective study, we reviewed the results of SFs of 93 cases of RA. All had a classical RA according to the ACR classification criteria [4], and $76 \%$ of them were rheumatoid factor positive. Synovial fluids were systematically examined for the presence of crystals. CPPD crystal deposition disease was diagnosed when typical rod-shaped, slightly positive birefringent crystals were observed under compensated polarised light at $40 \times$ magnification after centrifugation of SF. The presence of radiological signs of CPPD crystal deposition (chondrocalcinosis) was not mandatory to diagnosis and was not systematically recorded. In most cases, SF was obtained from knee joints. SF analysis included leucocyte count with a differential. Apatite crystals were not searched routinely. Demographic data of patients with and without CPPD crystal deposits in SF were compared. The number of lower limb joint prostheses was recorded at the time of SF sampling.

\section{Results}

Of the 93 RA cases, 64 were females (69\%). The mean age was 64.5 years (limits 23-89 years). CPPD crystal deposits were observed in SF in 24 of the 93 cases (25.8\%). Table 1 shows comparisons of duration of disease and number of prostheses of lower limbs for both groups. Duration of disease was quite long with a mean superior to 12 years, but with no statistically significant difference between both groups. Percentage of cases with a prosthesis was higher in the group with associated CPPD deposits (37.5 vs $21.7 \%$ ), representing an odd ratio of 2.2. Figure 1 illustrates one of the cases.

\section{Discussion}

Associated CPPD crystal deposition disease has been considered to be very rare in RA [3]. We found, by a systematic 
Table 1 Comparison of duration of disease and number of lower limb prosthesis by group

\begin{tabular}{|c|c|c|c|}
\hline & $\begin{array}{l}\mathrm{RA}+\mathrm{CPPD} \\
(n=24)\end{array}$ & $\begin{array}{l}\text { RA alone } \\
(n=69)\end{array}$ & \\
\hline $\begin{array}{l}\text { Duration of disease } \\
(\text { mean } \pm \text { SD) }\end{array}$ & $12.1 \pm 2.7$ & $7.8 \pm 1.5$ & NS* \\
\hline Women & $15 \pm 3.9$ & $8.9 \pm 1.5$ & NS* \\
\hline Men & $8 \pm 3.1$ & $5 \pm 1.2$ & NS* \\
\hline Prosthesis of lower & 9 & 15 & OR \\
\hline $\operatorname{limb}$ & & & 2.2 \\
\hline
\end{tabular}

$N S$ Not significant, $O R$ odds ratio

*By $t$ test

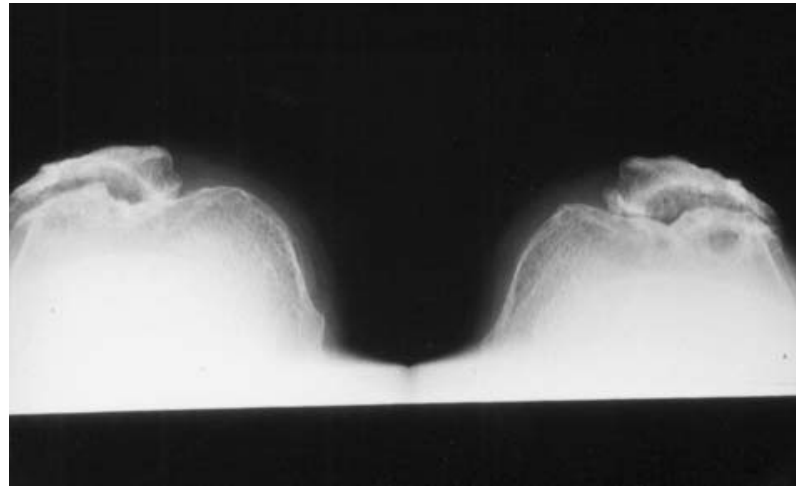

Fig. 1 Severe destructive arthropathy of the femoropatellar joint in a 75-year-old woman having a seropositive RA for 21 years. CPPD crystals were not found until January 2003

analysis of SF, that the prevalence of pyrophosphate crystals in RA knee joints is much higher than previously suspected. Previous studies on the incidence of pyrophosphate arthropathy associated to RA have been made mainly on the basis of radiographs, which could explain why the incidence was much lower than in our series [5]. Radiographic diagnosis of a superimposed chondrocalcinosis can be quite difficult in advanced RA. Finally, the use of centrifuged SF specimens, a technique that certainly improves the detection of CPPD crystals, could also explain this apparent discrepancy. Whether centrifugation was systematically performed in the other studies was not mentioned [6].

The fact that most SF were from knee joints may introduce a bias in our study. CPPD crystal deposits were found frequently in this RA population. Median RA duration of disease was superior to 12 years, and CPPD crystal deposits may just reflect accumulating joint damage overtime. It is well known that localized trauma predis- poses to dystrophic limited chondrocalcinosis, a phenomenon that could explains part of our high prevalence.

However, presence of CPPD crystal deposits was demonstrated in more than $25 \%$ of those SF, and they could also be a marker of a more severe outcome as suggested by a trend toward a higher number of prostheses in RA patients with coexistent crystals in SF. Calcium crystal deposits (CPPD or apatite) can be found in about $60 \%$ of patient with OA in a preoperative knee [7], and their role as innocent bystander or culprit is still debated. In RA patients, the question also remains and will not be answered by our study. However, the observed higher incidence of prostheses despite a not significantly different disease duration suggests that demonstration of CPPD crystals in SF of a RA joint can be regarded as predictive factor of impending joint destruction and surgery need. Further studies will be needed to define if CPPD crystals have any causative role in this ominous evolution.

\section{Take home message}

- CPPD crystals are found in a fourth of SF from longstanding RA patients, a percentage much more frequent than previously thought.

- Coexisting CPPD crystals in SF of RA patients is potentially a predictive factor of impending joint destruction and surgery need, an observation needing further studies.

\section{References}

1. Menkes CJ, Simon F, Delrieu F, Forest M, Delbarre F (1976) Destructive arthropathy in chondrocalcinosis articularis. Arthritis Rheum 19:329-348

2. Gerster JC, Vischer TL, Boussina I, Fallet GH (1975) Joint destruction and chondrocalcinosis in patients with generalised osteoarthrosis. Br Med J 2:684-685

3. Doherty M, Dieppe P, Watt I (1984) Low incidence of calcium pyrophosphate dihydrate crystal deposition in rheumatoid arthritis, with modification of radiographic features in coexistent disease. Arthritis Rheum 27:1002-1009

4. Arnett FC, Edwathy SM, Bloch DA et al (1988) The American Rheumatism Association 1987 revised criteria for the classification of rheumatoid arthritis. Arthritis Rheum 31:315-324

5. Sany J, Rosenberg F, Bataille R, Serre H (1977) Chondrocalcinoses secondaires et associées. Rev Rhum 44:565-577

6. Van Linthoudt D, Schumacher HR (1992) Frequency of synovial fluid (SF) crystals in different inflammatory rheumatic diseases. Arthritis Rheum Suppl 35:R9

7. Derfus BA, Kurian JB, Butler JJ et al (2002) The high prevalence of pathologic calcium crystals in pre-operative knees. J Rheumatol 29:570-574 